\title{
Mass loss in main-sequence B stars
}

\begin{abstract}
Jiří Krtička
Ústav teoretické fyziky a astrofyziky, Masarykova univerzita, 61137 Brno, Czech Republic

e-mail: krticka@physics.muni.cz

Received 28 May 2013 / Accepted 19 February 2014

ABSTRACT

We calculate radiatively driven wind models of main-sequence B stars and provide the wind mass-loss rates and terminal velocities. The main-sequence mass-loss rate strongly depends on the stellar effective temperature. For the hottest B stars the mass-loss rate amounts to $10^{-9} M_{\odot}$ year $^{-1}$, while for the cooler ones the mass-loss rate is lower by more than three orders of magnitude. Mainsequence B stars with solar abundance and effective temperatures lower than about $15000 \mathrm{~K}$ (later than spectral type B5) do not have any homogeneous line-driven wind. We predict the wind mass-loss rates for the solar chemical composition and for the modified abundance of heavier elements to study the winds of chemically peculiar stars. The mass-loss rate may either increase or decrease with increasing abundance, depending on the importance of the induced emerging flux redistribution. Stars with overabundant silicon may have homogeneous winds even below the solar abundance wind limit at $15000 \mathrm{~K}$. The winds of main-sequence B stars lie below the static limit, that is, a static atmosphere solution is also possible. This points to an important problem regarding the initiation of these winds. We discuss the implications of our models for rotational braking, filling the magnetosphere of Bp stars, and for chemically peculiar stars.
\end{abstract}

Key words. stars: winds, outflows - stars: mass-loss - stars: early-type - hydrodynamics

\section{Introduction}

Line-driven winds are most often studied in the most luminous hot stars. These stars have strong winds that are easy to observe and that significantly influence the stellar evolution. However, winds of less luminous stars, especially mainsequence B stars, also have considerable observational and theoretical consequences. Although the winds in these stars do not manifest themselves as prominent spectral features or have a decisive influence on the way the stars evolve, weaker winds affect a number of observational phenomena that gain considerable astrophysical attention.

Main-sequence B stars lie at the boundary between luminous $\mathrm{O}$ stars, where the radiative force expels huge amounts of mass from the star (Puls et al. 2008), and main-sequence A stars, where the minute effect of the radiative levitation horizontally redistributes the elements in easily recognizable surface spots (Michaud 2004). It has long been speculated that the outflow is a missing ingredient that is needed to quantitatively explain the resulting abundance anomalies. Such an explanation faces a strong problem because we lack consistent theoretical predictions of wind mass-loss rates at low luminosity.

Traditionally, two parameters are invoked to explain all aspects of the evolution of single stars: the initial mass and the chemical composition. However, it now seems that at least a third parameter is essential to explain the single-star evolution: the initial stellar angular momentum (Meynet et al. 2006). Consequently, rotation becomes one of the crucial parameters of evolution models. The stellar rotation may not only contribute to the internal mixing of chemical elements, but it may also bring a star near critical rotation (Ekström et al. 2012). Fast rotation is prerequisite for the collapsar model of gamma-ray bursts (Yoon et al. 2006). Unfortunately, the evolutionary time scale is so long that testing the predictions of rotational velocity evolution models directly is a forbidding task. However, there is one exception: the rotationally modulated light variations of chemically peculiar stars provide a powerful clock that can reveal even minute changes of the rotational period. Studies based on such a clock (Pyper et al. 1998; Mikulášek et al. 2011) have shown period variations that might be connected with angular-momentum loss via magnetized stellar winds. However, precise mass-loss rate predictions for these stars are lacking, therefore one cannot test the angular momentum loss hypothesis quantitatively.

As a result of its low density, the wind material might be trapped in the magnetospheres of Bp stars. Resulting magnetospheric clouds are the prime origin of the light variability of $\sigma$ Ori E (Landstreet \& Borra 1978; Townsend et al. 2005). Detailed properties of these clouds, such as the time scale of their build-up, are poorly constrained due to uncertain mass-loss rates at low luminosity.

Last but not least, winds of low-luminosity hot stars are also appealing theoretically. They provide a unique environment in which many astrophysical phenomena can be studied, including the particle collisions that lead to the multicomponent nature of the flow (e.g., Springmann \& Pauldrach 1992; Krtička et al. 2006), or the line Doppler-heating that influences the wind temperature (Gayley \& Owocki 1994). Note, however, that because the latter two effects occur in the outer wind, they typically do not affect the mass-loss rate.

Stellar winds of main-sequence B stars are difficult to study observationally. The $\mathrm{H} \alpha$ line, which is a crucial mass-loss-rate indicator in $\mathrm{O}$ stars, is unsuitable for determining a mass-loss rate of the order of $10^{-8} M_{\odot}$ year $^{-1}$ or lower (Puls et al. 2008), that is, in the domain of main-sequence B stars. Moreover, the mass-loss rate determination of main-sequence B stars from observations may be uncertain not only as the result of the effect of clumping on the line profiles (Sundqvist et al. 2011; Šurlan et al. 2012), but also as the result of the weak-wind problem 
(e.g., Bouret et al. 2003; Martins et al. 2005; Marcolino et al. 2009). The latter problem is connected with observed ultraviolet wind-line profiles in low-luminosity $\mathrm{O}$ stars, which are weaker than theory predicts. The weak mass-loss rates inferred from weak ultraviolet lines are (at least in one case) supported by the mass-loss rate derived from the $\mathrm{Br} \gamma$ line (Najarro et al. 2011).

On the other hand, there are independent observational indications (Huenemoerder et al. 2012, Gvaramadze et al. 2012) that show that the ultraviolet line profiles significantly underestimate the mass-loss rate in low-luminosity $\mathrm{O}$ stars. The weakness of the ultraviolet line profiles can be explained by the high wind temperature as a result of the shock heating in a medium with such a low density that is not able to cool down radiatively (Lucy \& White 1980; Cohen et al. 2008; Krtička \& Kubát 2009b; Lucy 2012). From this point of view the theoretical models provide a reliable choice for estimating B star mass-loss rates.

To improve the situation of poorly known wind parameters in main-sequence B stars, we here provide mass-loss rate estimates based on our own non-local thermodynamic equilibrium (NLTE) wind code.

\section{Description of models}

To predict main-sequence B star mass-loss rates we applied the NLTE wind models of Krtička \& Kubát (2010) with a comoving frame $(\mathrm{CMF})$ line force. Our wind models assume stationary and spherically symmetric flow. They enable us to self-consistently predict wind structure and wind parameters just from the basic stellar parameters, that is, the effective temperature, mass, radius, and chemical composition.

The ionization and excitation state of considered elements was derived from the statistical equilibrium (NLTE) equations. For this purpose we adopted a set of ionic models from the TLUSTY grid of model stellar atmospheres (Lanz \& Hubeny 2003, 2007), which was extended using the data from the Opacity and Iron Projects (Seaton et al. 1992; Hummer et al. 1993). For phosphorus we employed data described by Pauldrach et al. (2001).

The NLTE model atmosphere emergent fluxes, which serve as a lower boundary condition of the radiative transfer equation in the wind, are calculated by the TLUSTY code (Lanz \& Hubeny 2007) for the same stellar parameters (the effective temperature, surface gravity, and chemical composition) as the wind models.

The line radiative force was calculated using the solution of the spherically symmetric CMF radiative transfer equation (Mihalas et al. 1975). The corresponding line data were extracted from the VALD database (Piskunov et al. 1995; Kupka et al. 1999). We applied the electron thermal balance method (Kubát et al. 1999) to calculate the radiative cooling and heating. These terms were calculated with the occupation numbers derived from the statistical equilibrium equations.

The continuity equation, equation of motion, and energy equation were solved iteratively using the Newton-Raphson method. An accelerating outflow was assumed in the first step of iterations. As a result of iterations we obtained the wind density, velocity, and temperature structure that satisfy the hydrodynamical equations. We found no case where the derived solution (faster than the radiative-acoustic waves at outer edge, Abbott 1980) was not unique, that is, the final converged solution does not depend on a particular choice of initial conditions. We assumed homogeneous flow, that is, we neglect possible differences in velocities of individual components. This is legitimate here, because we are mainly interested in the mass-loss rates.
Table 1. Stellar parameters of the model grid.

\begin{tabular}{lcccccc}
\hline \hline Model & $\begin{array}{c}\text { Sp. } \\
\text { type }\end{array}$ & $\begin{array}{c}T_{\text {eff }} \\
{[\mathrm{K}]}\end{array}$ & $\begin{array}{c}M \\
{\left[M_{\odot}\right]}\end{array}$ & $\begin{array}{c}R_{*} \\
{\left[R_{\odot}\right]}\end{array}$ & $\log \left(L / L_{\odot}\right)$ & $\begin{array}{c}v_{\text {esc }} \\
{\left[\mathrm{km} \mathrm{s}^{-1}\right]}\end{array}$ \\
\hline T30 & B0 & 30000 & 14.73 & 5.84 & 4.39 & 960 \\
T28 & & 28000 & 12.69 & 5.34 & 4.20 & 940 \\
T26 & B1 & 26000 & 10.88 & 4.87 & 3.99 & 910 \\
T24 & & 24000 & 9.26 & 4.45 & 3.77 & 880 \\
T22 & & 22000 & 7.84 & 4.07 & 3.54 & 850 \\
T20 & & 20000 & 6.60 & 3.71 & 3.30 & 820 \\
T18 & & 18000 & 5.52 & 3.39 & 3.03 & 790 \\
T16 & B5 & 16000 & 4.58 & 3.09 & 2.75 & 750 \\
T14 & B6 & 14000 & 3.76 & 2.80 & 2.43 & 720 \\
\hline
\end{tabular}

The stellar parameters of the considered model grid are given in Table 1. Here we also provide the corresponding spectral types for selected models. The effective temperature $T_{\text {eff }}$ covers the range of earlier B stars, and the stellar mass $M$ and radius $R_{*}$ were calculated from the interpolation formulas derived for main-sequence stars by Harmanec (1988) with the effective temperature as a parameter. To calculate both wind and atmosphere models we assumed the solar chemical composition of Asplund et al. (2009). We also calculated additional models with a modified abundance of the elements that are most important for the line driving, that is, carbon, nitrogen, oxygen, silicon, sulfur, and iron. The range of considered abundances of these elements was partially motivated by the abundances typically found on the surface of chemically peculiar stars (e.g., Briquet et al. 2004; Lehmann et al. 2007; Bohlender et al. 2010).

\section{Mass-loss rates}

The predicted mass-loss rates $\dot{M}$ for B stars with solar metallicity in Table 2 nicely follow the $\mathrm{O}$ star mass-loss rate - luminosity relationship (Fig. 1). The mass-loss rates of solar metallicity B stars can be fitted via the formula

$\log \left(\frac{\dot{M}}{1 M_{\odot} \text { year }^{-1}}\right)=a+b\left(\frac{T_{\text {eff }}}{10^{4} \mathrm{~K}}\right)+c\left(\frac{T_{\text {eff }}}{10^{4} \mathrm{~K}}\right)^{2}$,

where

$a=-22.7, \quad b=8.96, \quad c=-1.42$.

The predicted mass-loss rates can be compared with the massloss-rate estimate corresponding to one optically thick line $\dot{M}=$ $8 \pi^{2} R_{*}^{2} v_{i j} H_{c}\left(v_{i j}\right) / c^{2}$ (Krtička \& Kubát 2009a, Eq. (11)). In this formula $H_{c}\left(v_{i j}\right)$ is the flux at the line frequency $v_{i j}$, but in Fig. 1 we use the maximum product of flux and frequency, giving the maximum mass-loss rate with one optically thick line. For B stars with higher luminosities this one-line mass-loss-rate estimate roughly corresponds to the predicted mass-loss rate because there are just a few lines that drive the wind. For stars with lower luminosities the one-line mass-loss-rate estimate overestimates the mass-loss rate because in these stars even the strongest lines become optically thin and their positions do not correspond to the maximum product $v H_{c}(v)$.

In main-sequence B stars the wind mass-loss rate significantly decreases with decreasing effective temperature (or luminosity). For a decrease of a factor of two in the effective temperature the decrease in mass-loss rates is more than three orders of magnitude. We were unable to find any wind solution for the solar metallicity model T14. This indicates that a homogeneous wind is not possible for $T_{\text {eff }} \lesssim 15000 \mathrm{~K}$. 
Table 2. Predicted mass-loss rates and terminal velocities for different abundances and different effective temperatures.

\begin{tabular}{|c|c|c|c|c|c|c|c|c|c|c|c|c|c|c|}
\hline \multirow[b]{2}{*}{ Model } & \multicolumn{2}{|c|}{ Solar } & \multicolumn{2}{|c|}{$[\mathrm{C} / \mathrm{H}]=-1$} & \multicolumn{2}{|c|}{$[\mathrm{C} / \mathrm{H}]=1$} & \multicolumn{2}{|c|}{$[\mathrm{N} / \mathrm{H}]=-1$} & \multicolumn{2}{|c|}{$[\mathrm{N} / \mathrm{H}]=1$} & \multicolumn{2}{|c|}{$[\mathrm{O} / \mathrm{H}]=-1$} & \multicolumn{2}{|c|}{$[\mathrm{O} / \mathrm{H}]=1$} \\
\hline & $\dot{M}$ & $v_{\infty}$ & $\dot{M}$ & $v_{\infty}$ & $\dot{M}$ & $v_{\infty}$ & $\dot{M}$ & $v_{\infty}$ & $\dot{M}$ & $v_{\infty}$ & $\dot{M}$ & $v_{\infty}$ & $\dot{M}$ & $v_{\infty}$ \\
\hline T30 & 210 & 3650 & 190 & 2280 & 210 & 5490 & 120 & 4010 & 370 & 4410 & 100 & 4110 & 190 & 4390 \\
\hline $\mathrm{T} 28$ & 160 & 4380 & 150 & 2620 & 110 & 8610 & 67 & 4540 & 160 & 5520 & 160 & 4500 & 130 & 4260 \\
\hline T26 & 96 & 3070 & 60 & 2400 & 60 & (2340) & 64 & (2440) & 90 & 4790 & 100 & 2990 & 79 & 3040 \\
\hline T24 & 39 & 1700 & 23 & 1720 & 42 & 1820 & 4.8 & 2040 & 38 & 3620 & 25 & 1850 & 39 & 1700 \\
\hline $\mathrm{T} 22$ & 7.9 & 1690 & 8.5 & 1580 & 14 & 1490 & 0.71 & 1760 & 14 & 2730 & 6.5 & 1820 & 12 & 1600 \\
\hline $\mathrm{T} 20$ & 3.4 & 1290 & 2.3 & 1250 & 1.5 & 1410 & 1.6 & 1260 & 6.4 & 1720 & 3.2 & 1300 & 3.6 & 1200 \\
\hline T18 & 0.91 & 820 & 0.42 & 460 & 0.58 & 900 & 0.51 & 760 & 1.8 & 1120 & 0.84 & 720 & 0.88 & 790 \\
\hline $\mathrm{T} 16$ & 0.072 & (240) & 0.023 & (180) & 0.06 & 520 & 0.038 & $(230)$ & 0.17 & 420 & 0.058 & $(240)$ & 0.06 & (230) \\
\hline \multirow[t]{2}{*}{$\mathrm{T} 14$} & \multicolumn{2}{|c|}{ no wind } & \multicolumn{2}{|c|}{ no wind } & \multicolumn{2}{|c|}{ no wind } & \multicolumn{2}{|c|}{ no wind } & \multicolumn{2}{|c|}{ no wind } & \multicolumn{2}{|c|}{ no wind } & \multicolumn{2}{|c|}{ no wind } \\
\hline & \multicolumn{2}{|c|}{$[\mathrm{Si} / \mathrm{H}]=1$} & \multicolumn{2}{|c|}{$[\mathrm{Si} / \mathrm{H}]=2$} & \multicolumn{2}{|c|}{$[\mathrm{Si} / \mathrm{H}]=3$} & \multicolumn{2}{|c|}{$[\mathrm{S} / \mathrm{H}]=-1$} & \multicolumn{2}{|c|}{$[\mathrm{S} / \mathrm{H}]=1$} & \multicolumn{2}{|c|}{$[\mathrm{Fe} / \mathrm{H}]=1$} & \multicolumn{2}{|c|}{$[\mathrm{Fe} / \mathrm{H}]=2$} \\
\hline Model & $\dot{M}$ & $v_{\infty}$ & $\dot{M}$ & $v_{\infty}$ & $\dot{M}$ & $v_{\infty}$ & $\dot{M}$ & $v_{\infty}$ & $\dot{M}$ & $v_{\infty}$ & $\dot{M}$ & $v_{\infty}$ & $\dot{M}$ & $v_{\infty}$ \\
\hline T30 & 250 & 4020 & 320 & 4490 & 490 & 2910 & 180 & 3720 & 380 & 3390 & 230 & 3040 & 1700 & 1720 \\
\hline $\mathrm{T} 28$ & 180 & 5510 & 210 & 5180 & 240 & 3200 & 130 & 4640 & 280 & 3850 & 140 & 3570 & 370 & 1910 \\
\hline $\mathrm{T} 26$ & 110 & (5270) & 150 & 3720 & 160 & 3070 & 83 & 3220 & 170 & 2820 & 83 & 3060 & 57 & 2390 \\
\hline $\mathrm{T} 24$ & 54 & 4580 & 81 & (1620) & 77 & 2450 & 29 & 1760 & 81 & 1710 & 31 & 1790 & 16 & 1740 \\
\hline $\mathrm{T} 22$ & 21 & 4020 & 23 & 6390 & 9.9 & 2250 & 5.3 & 1820 & 22 & 1730 & 6 & 1900 & 8.5 & 1690 \\
\hline T20 & 7.5 & 3630 & 8.4 & 7740 & 4.6 & 380 & 3 & 1270 & 4.5 & 1360 & 2.6 & 1320 & 1.8 & 1290 \\
\hline T18 & 3.1 & 1990 & 3.6 & 5270 & 1.9 & 510 & 0.84 & 790 & 1.4 & 830 & 0.74 & 830 & 0.39 & 790 \\
\hline $\mathrm{T} 16$ & 0.7 & 1400 & 0.7 & 2440 & 0.17 & (170) & 0.056 & $(240)$ & 0.085 & $(270)$ & 0.041 & (250) & & vind \\
\hline $\mathrm{T} 14$ & 0.01 & 650 & 0.004 & 1290 & 0.002 & 1550 & no & ind & no $\mathrm{v}$ & ind & no & vind & & vind \\
\hline
\end{tabular}

Notes. Mass-loss rates are given in units of $10^{-11} M_{\odot} \mathrm{year}^{-1}$. The terminal velocities $v_{\infty}$ are given in units of $\mathrm{km} \mathrm{s}^{-1}$. The terminal velocity in parentheses denotes a model for which it was not possible to calculate a monotonic solution to large radii (see the text).

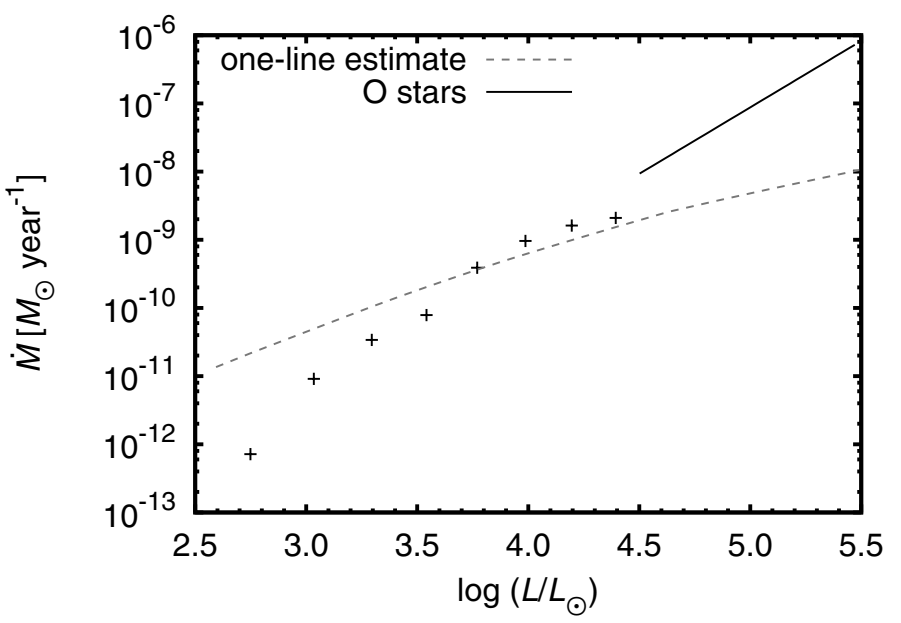

Fig. 1. Predicted mass-loss rates for solar metallicity as a function of the stellar luminosity. Overplotted (solid line) is the fit to main-sequence O star mass-loss rates (Krtička \& Kubát 2012). The dashed line denotes the one-line mass-loss rate estimate (Krtička \& Kubát 2009a).

In Table 3 we provide the radiative force multipliers (Castor et al. 1975; Abbott 1982) corresponding to our solar-metallicity models. The force multipliers describe the line distribution function (Puls et al. 2000), but in our approach we simply selected force multipliers that provided the best fit of wind mass-loss rate and terminal velocity derived in models with CMF line force. The parameter $\alpha$ decreases with the effective temperature to account for the decrease of the wind terminal velocity. To compensate for this the line force parameter $k$ increases on average with decreasing effective temperature. From this point of view the $\bar{Q}$ parameter of Gayley (1995) is advantageous, because its variations are much weaker in the considered stars, $\bar{Q} \approx 100$ (see Table 3, where we provide $\bar{Q}=\left[(1-\alpha) k\left(c / v_{\mathrm{th}}\right)^{\alpha}\right]^{1 /(1-\alpha)}$, where the fiducial hydrogen thermal speed is $\left.v_{\mathrm{th}}=\sqrt{2 k T_{\mathrm{eff}} / m_{\mathrm{H}}}\right)$.
Table 3. Line force multipliers for the models with solar metallicity.

\begin{tabular}{lccc}
\hline \hline Model & $k$ & $\alpha$ & $\bar{Q}$ \\
\hline T30 & 0.024 & 0.635 & 35 \\
T28 & 0.019 & 0.670 & 54 \\
T26 & 0.044 & 0.610 & 95 \\
T24 & 0.38 & 0.435 & 110 \\
T22 & 0.20 & 0.46 & 61 \\
T20 & 0.60 & 0.390 & 95 \\
T18 & 2.50 & 0.295 & 130 \\
T16 & 29.5 & 0.140 & 210 \\
\hline
\end{tabular}

There are not many mass-loss rate predictions that can be compared with Eq. (1). Vink et al. (2001) predicted a massloss roughly by a factor 2 higher for the T30 model, but the T30 model is slightly outside the model grid considered by them. Pauldrach et al. (2001) predicted a mass-loss rate for the model with $T_{\text {eff }}=30000 \mathrm{~K}$ that is higher by a factor of 4 than that of the T30 model, but for a radius larger by a factor of 2 . After correcting for the dependence of the mass-loss rate on radius (assuming a rough dependence $\dot{M} \sim L^{2}$, Krtička \& Kubát 2012), this gives a mass-loss rate that is lower by a factor of 5 . When we extrapolate the rates of Unglaub (2008), again outside their assumed grid, our predictions are about a factor of 5 higher for the model T30, while they are higher by about a factor of 3 for $T_{\text {eff }}=25000 \mathrm{~K}$. Finally, for $T_{\text {eff }}=30000 \mathrm{~K}$ our models predict roughly one eighth of the rates predicted by de Jager et al. (1988), while for $T_{\mathrm{eff}}=22000 \mathrm{~K}$ our results are nearly the same.

In Fig. 2 we plot the relative contribution of individual elements to the radiative force in models with solar chemical composition. The relative contribution is plotted at the critical point, where the mass-loss rate of our models is determined. The contribution of individual elements to the radiative force is a strong function of the effective temperature. Carbon and nitrogen dominate the radiative force in the hottest B stars with 


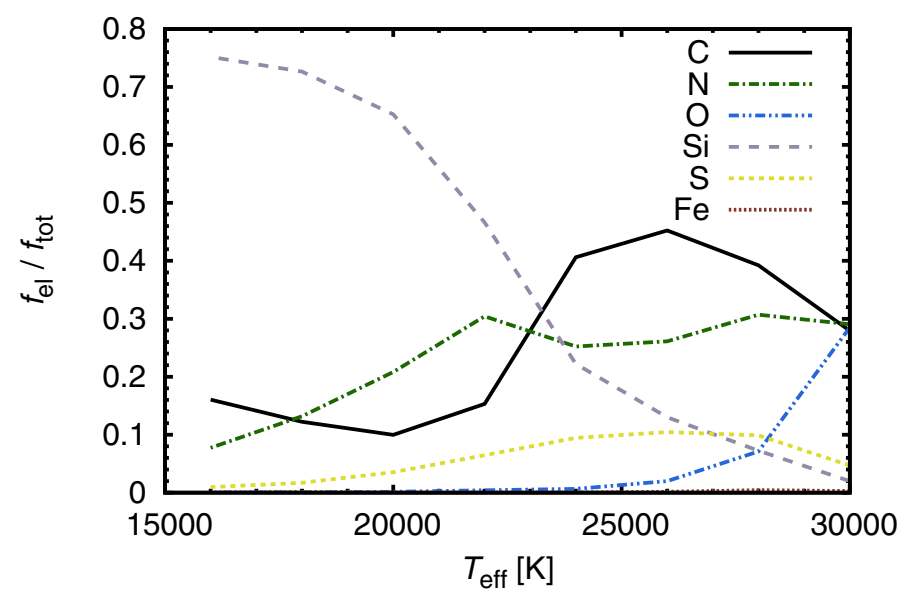

Fig. 2. Relative contribution of individual elements to the radiative force at the critical point as a function of the effective temperature. Models with solar abundance are plotted here. The contribution of iron is negligible.

$T_{\text {eff }} \approx 30000 \mathrm{~K}$, while in stars with $T_{\text {eff }} \lesssim 22000 \mathrm{~K}$ the lines of silicon dominate the radiative driving.

In contrast to $\mathrm{O}$ stars, there are only a few lines that drive the wind in B stars. Iron, which dominates at higher mass-loss rates (e.g., Vink et al. 2001), has a negligible contribution to the radiative force in $\mathrm{B}$ stars, because (as a result of its lower abundance compared to CNO elements) most of the iron lines become optically thin. However, this is not the only reason for a low contribution of iron to the radiative force in B stars, because iron has nearly the same abundance as silicon, which dominates the line driving in cooler B stars. In contrast to lighter elements such as silicon or carbon, iron ions do not have allowed transitions between the most populated ground level and excited levels with the lowest energies. Consequently, the iron resonance lines have wavelengths below the Lyman limit, where the stellar radiative flux is too low at given effective temperatures and does not contribute significantly to the radiative force.

A similar situation also occurs for oxygen, where the most abundant ion in B-star wind, O III, has the resonance lines in the Lyman continuum. On the other hand, carbon, nitrogen, and silicon have strong resonance lines in the Balmer continuum, therefore they significantly contribute to the radiative force. The importance of silicon is connected with the fact that the Si IV resonance lines lie close to the flux maximum. On the other hand, carbon is most significant at around $T_{\text {eff }}=25000 \mathrm{~K}$, where the relative contribution of resonance $\mathrm{C}$ IV lines at $1548 \AA$ and $1551 \AA$ is the strongest, while these lines become optically thin at lower effective temperatures because of the ionization shift from C IV to C III.

These results are also shown in Table 4, where we list the strongest lines that drive the wind in the region of the critical point. These lines can be used as potential wind indicators in B stars. On the other hand, their absence in the spectra does not mean that the wind does not exist, because we neglected other potentially important effects, for example, the wind X-ray ionization in the outer parts of the wind (e.g., Oskinova et al. 2011, see also Sect. 6.1).

It is common consensus that the mass-loss rate increases with increasing metallicity because the line absorption inceases. While this is true in most cases (e.g., Vink et al. 2001; Unglaub 2008), Fig. 3 shows that this is not always the case in the domain of main-sequence B stars. In several cases the mass-loss rate
Table 4. Strongest lines in the solar-metallicity models.

\begin{tabular}{lc}
\hline \hline Model & Lines (ions and wavelengths in A) \\
\hline T30 & C III 977, 1176; C IV 1548, 1551; N III 990, 992; \\
& N IV 765, 923; O III 833, 834, 835; O IV 788, 790; \\
& P V 1118, 1128; S V 786 \\
T28 & C III 977, 1176; C IV 1548, 1551; N III 990, 992; \\
& Si IV 1394, 1403; P V 1118; S IV 1063, 1073 \\
T26 & C III 977, 1176; C IV 1548, 1551; N III 990, 992; \\
& Si IV 1394, 1403; S IV 1063, 1073 \\
T24 & C III 977, 1176; C IV 1548, 1551; N III 990, 992; \\
& Si IV 1128, 1394, 1403; S IV 1063, 1073 \\
T22 & C III 977; C IV 1548, 1551; N III 990, 992; \\
& Si IV 1394, 1403; S IV 1063, 1073 \\
T20 & C III 977; N III 990, 992; Si IV 1394, 1403; S IV 1073 \\
T18 & C III 977; N III 990, 992; Si IV 1394, 1403 \\
T16 & C III 977; N III 990, 992; Si III 1207; Si IV 1394, 1403 \\
\hline
\end{tabular}

decreases with increasing abundance. This is caused by the influence of abundance on the emergent flux from the stellar atmosphere. Typically, the increase of abundance causes the flux redistribution from the far-ultraviolet part of the spectrum (where most of the flux is emitted) to the near ultraviolet and the visual part. If the redistribution is strong enough, it may affect the radiative force. We note that the same effect causes the light variability of chemically peculiar stars (e.g., Peterson 1970; Molnar 1973). Moreover, the stronger atmospheric lines with increasing abundance lower the line flux and hence the radiative force if these lines significantly contribute to the radiative driving (Babel 1996).

The increase of the carbon abundance causes the atmosphere flux redistribution from the wavelengths shorter than $1100 \AA$ to longer wavelengths, which in turn causes the decrease of the radiative force. Interestingly, the decrease of the carbon abundance also causes the decrease of the radiative force as a result of the decrease of the line absorption. This effect is apparent in models T16-T20 and T26-T30, whereas it is suppressed by stronger C IV lines in models T22 and T24 with overabundant carbon $[\mathrm{C} / \mathrm{H}]=1$. On the other hand, nitrogen, oxygen, and sulfur do not significantly influence the emergent atmosphere flux. Consequently, in this case the radiative force increases with increasing abundance of these elements. Silicon influences the emergent atmospheric flux for higher abundances $[\mathrm{Si} / \mathrm{H}] \gtrsim 2$. As a result, the mass-loss rate only strongly depends on the silicon abundance when the silicon is not significantly overabundant, that is, for $[\mathrm{Si} / \mathrm{H}] \lesssim 1$. Most interestingly, the mass-loss rate decreases with increasing iron abundance even if the iron does not contribute to the radiative force. This is again caused by the redistribution of the atmosphere radiative flux from the far-ultraviolet region to the near-ultraviolet and optical regions as a result of flux-blocking by numerous iron lines.

The increasing abundance of heavier elements (with the exception of silicon) does not help to find a wind solution for model T14. This is denoted as "no wind" in Table 2. Only stars enhanced by silicon can drive a homogeneous wind at $T_{\text {eff }}=$ $14000 \mathrm{~K}$, because silicon dominates the line force at this temperature.

The dependence of the mass-loss rate on the metallicity has significant consequences for chemically peculiar stars. The abundance of elements, most notably that of silicon and iron, 


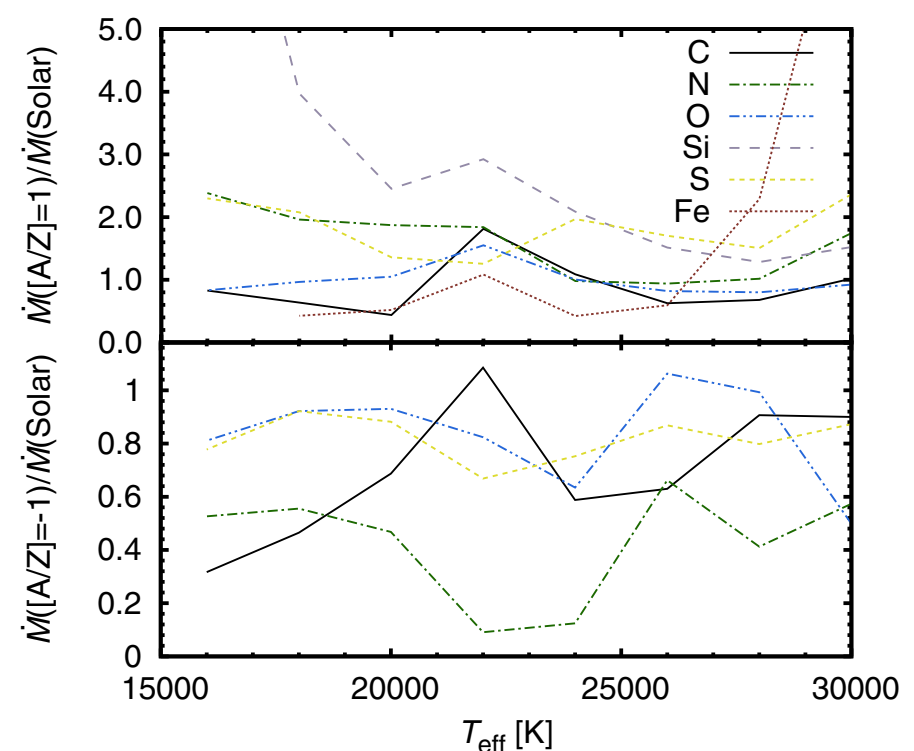

Fig. 3. Ratio of the mass-loss rate calculated with an abundance of a given element enhanced by a factor of ten (upper panel) and with an abundance reduced by a factor of ten (lower panel) to the mass-loss rate for a solar chemical composition as a function of stellar effective temperature.

varies with location on the stellar surface (e.g., Khokhlova et al. 2000; Lehmann et al. 2007). Consequently, the wind mass flux is variable across the surface. For helium-rich stars, where the abundances of silicon and helium are typically anticorellated, this means that the wind blowing from helium-rich regions is weaker than that from helium-poor ones. In magnetic stars the tilt of the flow under the influence of the magnetic field (Owocki \& ud-Doula 2004) also influences the mass-loss rate.

We also provide the wind terminal velocities $v_{\infty}$ derived from our models (see Table 2). The terminal velocities are sensitive to the ionization structure of the outer wind, possibly leading to the scatter of the observed $v_{\infty} / v_{\text {esc }}$ ratio in $\mathrm{O}$ stars (Lamers et al. 1995). This effect may explain some variations of $v_{\infty}$ seen in Table 2. Since in our case the winds are driven mostly by optically thick lines, the corresponding value of the effective line force parameter $\alpha \rightarrow 1$ indicates from simple scaling $v_{\infty} \sim \alpha /(1-\alpha) Z$ (Puls et al. 2000) an even stronger sensitivity of $v_{\infty}$ to the abundance. However, in many cases the wind may not reach this terminal velocity, either as a result of an inefficient X-ray cooling or because of multicomponent effects (see Sect. 6.1). This is also the reason why we argue that the discussion of terminal velocities that are lower than the escape speed $v_{\text {esc }}$ would be very premature in some cases (see Table 1 ).

In some cases the radiative force in the outer wind parts is too weak to maintain an accelerated wind flow. In this case a kink in the velocity profile occurs (Feldmeier \& Shlosman 2000) and wind velocity decreases in the outer parts of the wind (see Fig. 4). For these the terminal velocity in Table 2 corresponds to the highest wind velocity and is given in parentheses. This typically occurs in the wind models T16. For these the wind velocity is lower than the escape velocity $v_{\text {esc }}$ (see Table 1 ), and the wind may never leave the star. We note that our CMF procedure allows calculating the radiative force in an accelerating wind only, consequently, the models in Fig. 4 were derived assuming a Sobolev line force with $\mathrm{CMF}$ correction.

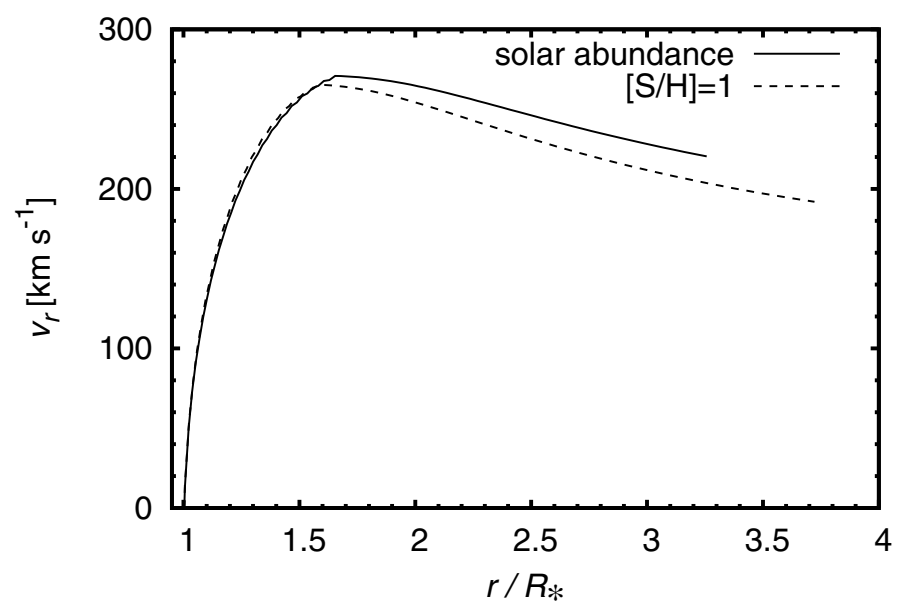

Fig. 4. Dependence of the radial wind velocity on radius in selected T16 wind models with a kink for a model with solar chemical composition (solid line) and with $[\mathrm{S} / \mathrm{H}]=1$ (dashed line).

\section{Wind limit at $T_{\text {eff }} \approx 15000 \mathrm{~K}$}

We were unable to find a wind solution for stars with $T_{\text {eff }}<$ $16000 \mathrm{~K}$ (except for the silicon-rich ones). This indicates that these stars have no wind. Therefore, we provide additional tests for these stars and compare the radiative force with the gravity. The winds are only possible when the magnitude of the radiative acceleration $g^{\mathrm{rad}}$ is larger than the magnitude of the gravity acceleration $g$,

$g^{\mathrm{rad}}>g$.

To enable the calculation of the radiative force even when there is no wind, the hydrodynamical variables (velocity, density, and temperature) were kept fixed during the tests. The velocity structure of our test models is given by an artificial velocity law

$v(r)=10^{-3} \sqrt{\frac{5}{3} \frac{k T_{\mathrm{eff}}}{m_{\mathrm{H}}}}+2 \times 10^{8} \mathrm{~cm} \mathrm{~s}^{-1} \frac{r-R_{*}}{R_{*}}$,

where $T_{\text {eff }}$ is the stellar effective temperature, $R_{*}$ the stellar radius, and $m_{\mathrm{H}}$ the mass of the hydrogen atom. The selection of such a specific velocity law does not significantly influence our results. The density structure is obtained from the equation of continuity. In these test models we assumed a constant wind temperature $\frac{2}{3} T_{\text {eff }}$, and the electron density was consistently calculated from the ionization balance. Since for these wind tests we did not solve the equation of motion, it was necessary to specify the wind mass-loss rate for which the wind existence was tested. For each set of stellar parameters these mass-loss rates are $10^{-12} M_{\odot}$ year $^{-1}, 10^{-13} M_{\odot}$ year $^{-1}, 10^{-14} M_{\odot}$ year $^{-1}$, and $10^{-15} M_{\odot}$ year $^{-1}$.

The wind tests support our expectations from the previous section. The wind is possible for stars with $T_{\text {eff }} \geq 16000 \mathrm{~K}$, whereas the homogeneous winds exist only for silicon-rich stars with $T_{\text {eff }}=14000 \mathrm{~K}$. From this we conclude that main-sequence stars with $T_{\text {eff }} \lesssim 15000 \mathrm{~K}$ without an enhanced silicon abundance have no homogeneous winds (i.e., winds composed of hydrogen, helium, and heavier elements).

We failed to find a wind solution for the T16 model with overabundant iron $[\mathrm{Fe} / \mathrm{H}]=2$. The test using the wind condition Eq. (3) showed that the radiative force is unable to drive wind close to the star in a slightly supersonic region as a result of strong flux redistribution. We also assumed that there is no homogeneous wind in this case. 
Abbott (1979) distinguished two wind limits in the H-R diagram. In stars above the static limit the wind can be self-initiated, that is, the radiative force is so strong that a static atmosphere is not possible. All these stars are expected to have winds. In stars below the static limit and above the wind limit the wind can be maintained if it already exists. These stars may or may not have winds, depending on initial conditions. The results of model atmosphere calculations show that all model stars lie below the static limit. The derived threshold at $T_{\text {eff }} \approx 15000 \mathrm{~K}$ corresponds to the wind limit. In main-sequence stars our detected wind limit is higher by roughly $2000 \mathrm{~K}$ than that derived by Abbott (1979), most likely as a result of more realistic model atmosphere fluxes. Stars below the wind limit may still have a purely metallic wind (Babel 1995).

\section{Observational consequences}

\subsection{Comparison of UV line profiles}

The comparison of observed and predicted wind line profiles would be the most natural test of the derived mass-loss rates. However, this can be complicated in the domain of mainsequence B stars studied here. In addition to the clumping problem in more luminous hot stars (e.g., Sundqvist et al. 2011; Šrlan et al. 2012) the weak-wind problem may also complicate the situation in main-sequence B stars. Consequently, we here also discuss indirect mass-loss rate indicators and postpone a detailed study of wind line profiles to a subsequent study.

We note that some studies concentrated on a detailed description of the line profiles that might be contributed to the winds, instead of on the wind parameter determination. In some cases this might be a safer option, because resonance lines may originate both in the wind and in the atmosphere. Detailed atmosphere models are necessary to judge whether the line originates in the wind or in the atmosphere (Hubený et al. 1986). Slettebak (1994) concluded that Si IV absorption line profiles are present up to a spectral type of B8 $\left(T_{\text {eff }}=11600 \mathrm{~K}\right)$, while he found that Si IV edge velocities and equivalent widths do not vary significantly with spectral type for stars later than about B5 $\left(T_{\text {eff }}=15500 \mathrm{~K}\right.$, see Figs. 4 and 10 therein). This roughly agrees with the wind limit at $T_{\text {eff }} \approx 15000 \mathrm{~K}$ detected by us. The C IV lines are visible up to a spectral type of B3 $\left(T_{\text {eff }}=19100\right)$ in standard (i.e., non-Be) stars, in agreement with their negligible contribution to the radiative force in these stars (see Table 4). The observed variations of the Si IV (or C IV) resonance line profiles may be tentatively explained if in stars of earlier spectral type than B5 (or B3 in the case of C IV lines) we observe absorption caused by the wind, while in the latter ones the absorption in Si IV lines originates in the atmosphere.

\subsection{Mass-loss rates of magnetic $B$ stars}

Oskinova et al. (2011) derived the mass-loss rate for magnetic B-type stars from the ultraviolet line profiles taking into account the X-ray emission. This could be crucial in the B-star domain (similar to late O dwarfs, Martins et al. 2005) because of the strong influence of X-rays on the wind ionization equilibrium. Oskinova et al. (2011) provided two mass-loss rate estimates derived from C IV and Si IV lines. These estimates differ by up to 1 dex, probably as a result of uncertain wind ionization state.

Here we selected the mass-loss rates derived from the Si IV lines, because these lines always give a higher mass-loss rate. The weaker C IV lines can be explained for instance by a lower ionization fraction of C IV than derived in the models. We
Table 5. Comparison of mass-loss rates derived by Oskinova et al. (2011) from Si IV lines and the rates predicted from the stellar effective temperature using Eq. (1).

\begin{tabular}{lccc}
\hline \hline Star & $T_{\text {eff }}[\mathrm{K}]$ & \multicolumn{2}{c}{$\log \left(\dot{M} / 1 M_{\odot}\right.$ year $\left.^{-1}\right)$} \\
& & Oskinova et al. & Prediction Eq. (1) \\
\hline$\tau$ Sco & 30700 & -8.6 & -8.6 \\
$\beta$ Cep & 25100 & -9.1 & -9.2 \\
$\xi^{1}$ CMa & 27000 & -10 & -8.9 \\
V2052 Oph & 23000 & -9.7 & -9.6 \\
$\zeta$ Cas & 20900 & -9.7 & -10.2 \\
\hline
\end{tabular}

note that the opposite (lower mass-loss rate with stronger Si IV lines) would be more difficult to explain. In Table 5 we compare these mass-loss rates derived from observations with predicted rates derived using Eq. (1) for the effective temperature given in Oskinova et al. (2011, see also Table 5) for the studied magnetic stars. The mass-loss rates derived from observations and the predicted rates differ in most cases by no more than $0.1 \mathrm{dex}$, consequently, we conclude that there in this case observation and theory agree well.

The $\mathrm{H} \alpha$ emission from the material trapped in the corotating magnetosphere is typically observable for the stars with effective temperature higher than about $16000 \mathrm{~K}$ (Zboril et al. 1997, Petit et al. 2013). The magnetosphere is filled by the stellar wind, therefore this anticipated effective temperature limit for stars with magnetospheric $\mathrm{H} \alpha$ emission may be explained by the disappearance of homogeneous wind at $T_{\text {eff }} \approx 15000 \mathrm{~K}$.

\subsection{Rotational braking of Bp stars}

The helium-rich star $\sigma$ Ori $\mathrm{E}$ shows rotational braking (Townsend et al. 2010) that can be explained to be the result of angular momentum loss by a magnetized wind. The spin-down time depends on the stellar parameters, polar magnetic field strength $B_{\mathrm{p}}$, and on the wind parameters, $\tau_{\text {spin }} \sim$ $M\left(v_{\infty} / \dot{M}\right)^{1 / 2} /\left(B_{\mathrm{p}} R_{*}\right)$ (ud-Doula et al. 2009).

For $\sigma$ Ori $\mathrm{E}$ we adopt the stellar parameters from Hunger et al. $\left(1989, T_{\text {eff }}=22500 \mathrm{~K}, M=8.9 M_{\odot}, R_{*}=5.3 R_{\odot}\right)$. For this star we derive from Eq. (1) corrected for the assumed radius via $\dot{M} \sim L^{2} \sim R^{4}$ (Krtička \& Kubát 2012) the mass-loss rate $\dot{M}=5.5 \times 10^{-10} M_{\odot}$ year $^{-1}$. This is a significantly lower value than that derived by Krtička et al. (2006) as a result of the use of model atmospheres with metal opacity and CMF line force in the wind models.

With the polar field strength $B_{\mathrm{p}}=9.6 \mathrm{kG}$, moment of inertia constant $k=0.05$ (Meynet \& Maeder 2006), and the terminal velocity $v_{\infty}=1690 \mathrm{~km} \mathrm{~s}^{-1}$ (see Table 2), we derive from Eq. (25) of ud-Doula et al. (2009) a spin-down time of 1.7 Myr. This agrees well with the value of $1.34 \mathrm{Myr}$ derived from photometry (Townsend et al. 2010).

The rotation braking as the result of angular-momentum loss via the magnetized wind is effective in stars with a wind and a magnetic field. In Fig. 5 we plot the dependence of the spindown time (ud-Doula et al. 2009) for the solar abundance models studied here for three different values of the polar strength of the dipolar field. The spin-down time is nearly constant for stars hotter than about $T_{\text {eff }}=23000 \mathrm{~K}$. At this temperature range the decrease of the mass-loss rate with decreasing temperature is compensated by the decrease of the $M / R_{*}$ ratio and $v_{\infty}$. At lower effective temperatures the spin-down time significantly increases with decreasing temperature as a result of the decreasing mass-loss rate. 


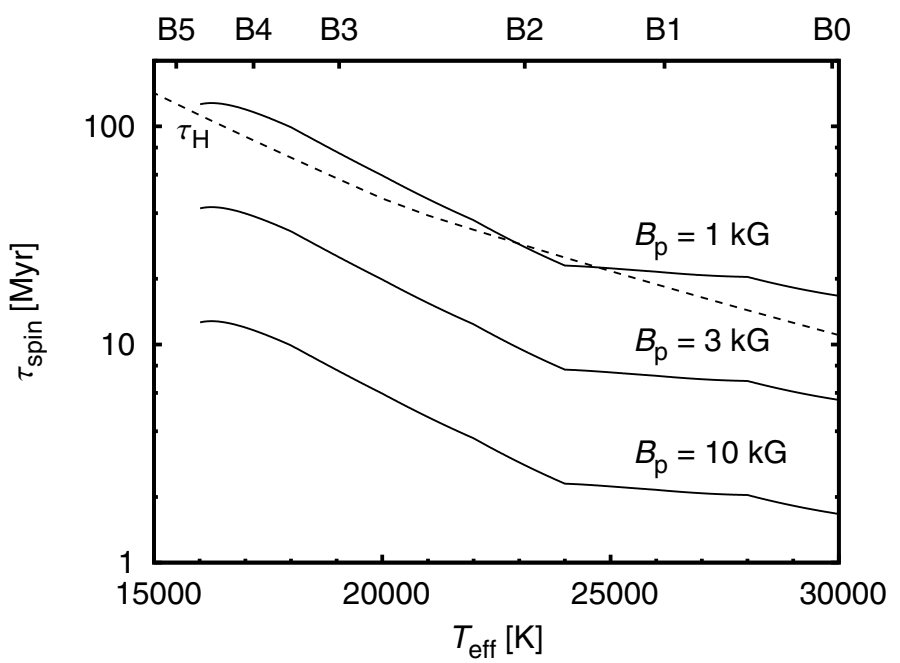

Fig. 5. Spin-down time as a result of angular momentum loss via magnetized stellar wind as a function of effective temperature for different polar field strengths. Overplotted is the main-sequence lifetime $\tau_{\mathrm{H}}$ derived from the non-rotating models of Ekström et al. (2012).

With long-term observation series of individual stars the rotation braking with the spin-down time up to roughly $10 \mathrm{Myr}$ can be detected (Mikulášek et al. 2011). According to Fig. 5 this means that rotational braking is detectable in stars with effective temperature $T_{\text {eff }} \gtrsim 22000 \mathrm{~K}$ and a medium-strong field, while in cooler stars only a very strong magnetic field causes detectable period changes. For stars with a polar field intensity stronger than about $2 \mathrm{kG}$ the spin-down time is shorter than the mainsequence lifetime (see Fig. 5). Consequently, the population of stars with strong magnetic field should on average show slower rotation than stars with a weaker field.

These results can be compared with wind-braking in nonmagnetic stars. The time derivative of the stellar angular momentum in the case of rotational braking of a uniformly rotating star can be expressed as $\dot{J}=\eta M R^{2} \dot{\Omega}$, where $\eta$ is a dimensionless constant given by the mass distribution in the star, and $\dot{\Omega}$ is the time derivative of the angular velocity $\Omega$. With angularmomentum loss via a nonmagnetized wind $\dot{J}=\frac{2}{3} \dot{M} R^{2} \Omega$ (here $\frac{2}{3}$ comes from integrating over the spherical surface) the spin-down time is $\tau_{\text {wind }} \approx \Omega / \dot{\Omega}=\frac{3}{2} \eta M / \dot{M}$. With $\eta=0.05$ (which is appropriate for a star of mass $M=9 M_{\odot}$, Meynet $\&$ Maeder 2006), the spin-down time is higher by at least one order of magnitude than the main-sequence lifetime. Consequently, rotational braking by a nonmagnetized wind is negligible in main-sequence B stars.

\section{Discussion}

\subsection{Model simplifications}

Many effects are neglected in our models. The X-ray emission strongly influences the ionization equilibrium at low effective temperatures (Martins et al. 2005). However, our models show that the X-ray emission does not influence the radiative force close to the star where the mass-loss rate is determined (Krtička \& Kubát 2009b). This is supported by Oskinova et al. (2011), who showed that the observed level of X-ray emission does not lead to this decrease of the radiative force and mass-loss rates that would explain the problem with too weak wind line profiles in low-luminosity stars (weak-wind problem, e.g., Bouret et al. 2003; Martins et al. 2005).

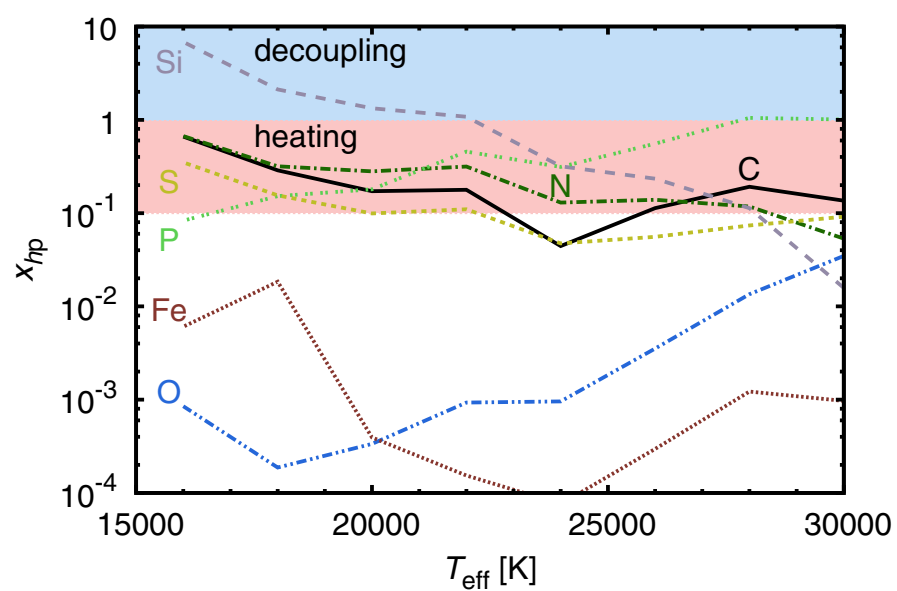

Fig. 6. Variations of the maximum relative velocity difference of individual elements and protons as a function of stellar effective temperature in models with solar chemical composition.

Our predicted wind mass-loss rates may be affected by a weak-wind problem. However, this is probably not the case, because the weak-wind problem may be caused by a too long cooling length in radiative shocks in the wind (Lucy \& White 1980; Cohen et al. 2008; Krtička \& Kubát 2009b; Lucy 2012). Such shocks occur well above the critical point (Owocki et al. 1988; Feldmeier et al. 1997), and consequently do not influence the mass-loss rate. This would mean that the models predict correct mass-loss rates, but the mass-loss rates derived from the line profiles are too low as a result of the X-ray overionization. However, this explanation still lacks a detailed proof using timedependent hydrodynamical models that take shock heating into account (e.g., Feldmeier et al. 1997). Spectra synthesized using such time-dependent models then should show weak-wind lines that can be compared with the observed lines as a final test of this scenario.

Stellar winds of hot stars have a multicomponent nature. Mostly heavier ions and free electrons are accelerated as a result of absorption of stellar radiation. The momentum acquired by these components is transferred to hydrogen and helium via Coulomb collisions. The wind can be treated as a onecomponent flow at high wind densities, while at low wind densities typical for main-sequence B star winds the effects connected with the multicomponent flow are significant. However, either frictional heating (Springmann \& Pauldrach 1992; Krtička et al. 2006) or the decoupling of wind components (Owocki \& Puls 2002; Votruba et al. 2007) typically occur at velocities higher than the critical one, therefore they do not affect the wind mass-loss rate, but may affect the terminal velocity.

The importance of the multicomponent effects is given by a relative velocity difference $x_{h \mathrm{p}}$ (Eq. (18) in Krtička 2006) between a given element and protons. For $x_{h p} \lesssim 0.1$ the multicomponent effects are unimportant, for $x_{h \mathrm{p}} \gtrsim 0.1$ the frictional heating typically influences the wind temperature, and for $x_{h \mathrm{p}} \gtrsim 1$ decoupling of components is possible. We calculated the relative velocity difference following Krtička (2006) for all considered elements in all models. The relative velocity difference between wind components increases with radius as a result of the decreaseing wind density. In all models presented here (except for the T14 silicon-rich models) the relative velocity difference is lower than 1 at the critical point, therefore the decoupling does not affect the mass-loss rate, which is determined at the critical point. 
In some models the multicomponent effects may influence the terminal velocity. This is documented in Fig. 6, where we plot the maximum relative velocity difference $x_{h \mathrm{p}}$ between a given element and protons found in the wind. For the hottest stars $T_{\text {eff }} \gtrsim 28000 \mathrm{~K}$ phosphorus may decouple from the wind. However, this probably does not significantly affect the wind, because the contribution of phosphorus to the radiative force is negligible. For stars with $T_{\text {eff }} \gtrsim 24000 \mathrm{~K}$ the multicomponent nature of the flow leads to additional heating due to carbon, nitrogen, and silicon. For cooler stars with $T_{\text {eff }} \lesssim 22000 \mathrm{~K}$ the decoupling of elements may occur in the outer parts of the wind. For these stars the terminal velocities in Table 2 needs to be corrected for multicomponent effects.

In $\mathrm{O}$ stars the winds are typically relatively dense and the stars themselves are luminous, which makes the typical time needed for the ionization or recombination very short, in most cases much shorter than the typical flow-time. As a result, the advection term in NLTE equations can be safely neglected (Lamers $\&$ Morton 1976). This is no longer true in B stars (Oskinova et al. 2011) with their weaker radiation field and thinner winds. We compared the ionization and recombination rates of our model ions with the inverse of the flow time $v / r$, where $v$ is the radial velocity. From our models it follows that for stars with $T_{\text {eff }} \gtrsim 20000 \mathrm{~K}$ the advection term for dominant ions is negligible in the inner parts of the wind where the mass-loss rate is determined. The relative unimportance of advection term for the dominant ionization states (that drive the wind) follows from the fact that the ionization from lower ionization state is fast, while the corresponding recombination is slow, and the opposite is true for the higher ionization state. On the other hand, in these stars the advection term may affect the population of minor ionization states and even also of dominant ones in the outer wind regions, where the lines used as observational indicators originate (Martins et al. 2005). For stars cooler than about $T_{\text {eff }}=20000 \mathrm{~K}$ the radiative processes become slower than the wind radial expansion, and in turn the advection term may become important even for the dominant ions close to the critical point. For these stars the ionization equilibrium becomes partially frozen in the flow and the advection term may affect the wind mass-loss rate.

\subsection{Wind initiation}

The calculated model atmospheres show that the radiative force in the static model atmospheres in main-sequence B stars does not overcome the gravity (up to $\tau_{\text {ross }} \approx 10^{-7}$ ). This means that all model stars lie below the static limit introduced by Abbott (1979). We have shown that two different solutions are possible, that is, a static atmosphere and an extended atmosphere with wind. We assumed that the wind was already initiated. It is unclear how the winds are initiated in this case, or even if they are initiated at all. There are several possibilities how the winds might be initiated. The radiative force on individual heavier elements overcomes the gravity in all models. This means that a metallic wind can be initiated first from the uppermost parts of the stellar atmosphere, which subsequently also drives hydrogen and helium out from the stellar atmosphere. The other possibility is that a random perturbation in the stellar atmosphere de-shadows the line from the photospheric line profile, leading to the initiation of an outflow. Winds may also be initiated in the pre-main-sequence phase, when the star has a lower surface gravity. The wind initiation is an interesting problem on its own, which deserves further study.

\subsection{Filling the magnetosphere of $\sigma$ Ori $E$}

There are few Bp stars (including $\sigma$ Ori E) that show light variability as a result of light absorption in the circumstellar material trapped thanks to the interplay between magnetic field and centrifugal force. The amount of the light variability enables us to estimate the mass of the magnetosphere and (with the wind mass-loss rate) the time needed to fill the clouds.

The amplitude of the $\sigma$ Ori $\mathrm{E}$ light variability is about $0.2 \mathrm{mag}$, which means that about $20 \%$ of the stellar flux is absorbed by the clouds, implying the minimum optical depth of the clouds is $\tau=0.2$. If the magnetosphere has the same opacity as the atmosphere, then the mass of the magnetosphere can be estimated as the column mass of the atmosphere $m(\tau=0.2)$ at the optical depth $\tau=0.2$ multiplied by the stellar surface area. From the model atmosphere of $\sigma$ Ori $\mathrm{E}$ we derive $m(\tau=0.2)=0.1 \mathrm{~g} \mathrm{~cm}^{-2}$, giving the total mass of $\sigma$ Ori E magnetosphere $M_{\mathrm{m}}=4 \pi R_{*}^{2} m(\tau=0.2) \approx 10^{-10} M_{\odot}$. This should be regarded as a minimum mass, because the material could be optically thick, which would mean that the optical depth were significantly higher.

Another estimate of the mass of the magnetosphere can be derived by assuming that the light scattering on free electrons dominates the opacity. In the ionized medium the opacity cannot be lower than that given by the light scattering on free electrons. Then the absorbing column mass $m_{\mathrm{e}}=\tau m_{\mathrm{H}} / \sigma_{\mathrm{Th}}$, where $\sigma_{\mathrm{Th}}$ is the Thomson scattering cross-section, and $m_{\mathrm{H}}$ the hydrogen mass. This gives another estimate of the magnetospheric mass, $M_{\mathrm{m}} \approx 4 \pi R_{*}^{2} m_{\mathrm{e}}=4 \times 10^{-10} M_{\odot}$.

From the mass-loss rate of $\sigma$ Ori E given in Sect. 5.3 we can conclude that the shortest typical time needed to fill the magnetosphere of a star similar to $\sigma$ Ori $\mathrm{E}$ is about one year. This is shorter by two orders of magnitude than the breakout time of the magnetosphere estimated by Townsend \& Owocki (2005). From this we can conclude that many other Bp stars should show clear signatures of the matter trapped in the magnetosphere. However, this is not the case because such stars are very rare. Therefore we can infer that there has to be some as yet unknown process that incessantly removes the material deposited by the winds from the magnetospheres of most Bp stars (Neiner et al. 2012).

\subsection{How are wind and peculiarity connected?}

Some B and A stars show chemical peculiarity caused by a radiative diffusion (see, e.g., Romanyuk 2007, for a review). The slow motion caused by the wind in the atmosphere interacts with diffusion and may modify the observed abundance anomalies. The interplay between winds and peculiarity depends on the wind mass-loss rate, and the result is therefore different for stars with different effective temperatures or spectral types.

In helium-rich stars a very weak wind with a mass-loss rate of the order of $10^{-13}-10^{-12} M_{\odot}$ year $^{-1}$ is required to explain the overabundance of helium (Vauclair 1975; Vauclair et al. 1991). Typically, helium-rich stars span a temperature range of about $T_{\text {eff }}=18000-23000 \mathrm{~K}$ (Zboril et al. 1997; Hunger \& Groote 1999). For stars in this temperature range the solar abundance mass-loss-rate predictions give a mass-loss rate higher by one to two orders of magnitude than that required by a diffusion theory. Such a large mass-loss would destroy any chemical peculiarity (Vick et al. 2011). On the other hand, helium-rich regions typically are metal-poor (Khokhlova et al. 2000; Bohlender et al. 2010), implying a lower wind mass-loss rate. It seems that the helium overabundance may be maintained if it already exists, but it is not clear how the atmosphere was able to evolve to this 
state. Possibly the line-driven wind mass-loss rate increases as the effective temperature increases during star formation (Palla \& Stahler 1992), leading to helium overabundance at the time of appropriate mass-loss rate. We also note that in the presence of the magnetic field the surface mass-flux $\dot{m}$ is proportional to the tilt $\theta_{B}$ of the wind with respect to the local vertical direction, $\dot{m} \sim \cos ^{2} \theta_{B}$ (Owocki \& ud-Doula 2004). This might be another reason for the decreasing wind mass-loss rate even to values required by the diffusion theory in stars with overabundant helium.

In chemically peculiar stars with low effective temperatures $\left(T_{\text {eff }} \leq 15000 \mathrm{~K}\right)$ the interplay of peculiarity and winds with mass-loss rates of the order of $10^{-14}-10^{-12} M_{\odot}$ year $^{-1}$ should lead to observable overabundances of oxygen or neon (Landstreet et al. 1998). Oxygen overabundances are not observed, possibly implying the absence of such winds in these stars. This agrees with our finding that stars in this temperature range have no homogeneous line-driven wind. On the other hand, Michaud et al. (2011) argued that some mixing is required to explain the observed abundances in Sirius A, possibly with the stellar wind with mass-loss rates $10^{-13} M_{\odot}$ year $^{-1}$, which is not expected from our models. These stars could have pure metallic wind with mass-loss rates lower by about three orders of magnitude (Babel 1995), which is too weak to cause substantial mixing. Possibly, the required mixing is caused by the turbulence.

Despite this qualitative agreement, some open questions remain. For example, the chemically peculiar star CU Vir shows pulsed radio emission (Trigilio et al. 2000; Kellett et al. 2007), which requires a wind mass-loss rate of about $10^{-12} M_{\odot}$ year $^{-1}$ (Leto et al. 2006). However, stars with an effective temperature $T_{\text {eff }}=13000 \mathrm{~K}$ of CU Vir (Kuschnig et al. 1999) can have a purely metallic wind with a mass-loss rate lower by about three magnitudes (Babel 1995). It is unclear how this disagreement can be solved. The wind might originate from silicon-rich parts of CU Vir surface, but the predicted mass-loss rate (see Table 2) is still too low by at least one order of magnitude.

Chemically peculiar stars typically display an inhomogeneous surface distribution of elements with relative elemental abundance differences reaching a few orders of magnitude (e.g., Briquet et al. 2004; Lehmann et al. 2007; Bohlender et al. 2010). As a result of the dependence of the mass-loss rates on abundance (see Table 2), the mass flux from individual surface elements varies with location on the stellar surface. Moreover, the tilting of the flow in the presence of the magnetic field (Owocki $\&$ ud-Doula 2004) and possibly also the Zeeman effect influence the mass-loss rate. These effects may contribute to the observed rotational variability of the strength of ultraviolet resonance lines in Bp stars (Shore \& Brown 1990).

\subsection{Winds of fast-rotating stars}

The B star mass-loss rates are very sensitive to the stellar effective temperature. For fast-rotating stars where the gravity darkening becomes important this leads to a high ratio of polar to equatorial mass-loss rate and density. Taking as an example the star HR 7355 that rotates at about $90 \%$ of its critical rotational velocity (Rivinius et al. 2013) with a polar effective temperature of $19800 \mathrm{~K}$ and an equatorial temperature of $15700 \mathrm{~K}$, the mass-loss rate formula Eq. (1) yields the polar to equatorial mass-loss-rate ratio of about 40 . Here we neglected the nonradial component of the radiative force (Owocki et al. 1996), but this result still shows that the wind asymmetries in fast-rotating main-sequence B stars can be enormous.

\section{Conclusions}

We calculated NLTE radiatively driven wind models of mainsequence B stars with the CMF line force. We provided the wind mass-loss-rate predictions as a function of the stellar effective temperature. The early-B stars have line driven winds with massloss rates of the order of $10^{-9} M_{\odot}$ year $^{-1}$. For the cooler stars the mass-loss rate strongly decreases with their effective temperature, which means that the solar-metallicity main-sequence B stars with effective temperatures lower than about $15000 \mathrm{~K}$ have no homogeneous line-driven wind. This general picture qualitatively agrees with observations of UV resonance lines and with recent B star mass-loss-rate determinations.

Winds of main-sequence B stars are driven mainly by carbon, nitrogen, and silicon. In the winds of stars with $T_{\text {eff }} \lesssim$ $22000 \mathrm{~K}$ the silicon-driving dominates.

The mass-loss rates depend on the elemental abundances. For elements that do not significantly alter the emergent model atmosphere flux the mass-loss rate increases with increasing abundance. However, for elements that significantly influence the emergent flux (most notably iron) the mass-loss rate may decrease with increasing abundance of a given element. The dependence of the radiative force on the silicon abundance in the coolest stars studied here is so strong that wind may be possible even below the solar-abundance wind limit at $T_{\text {eff }}=15000 \mathrm{~K}$.

We discussed the implications of our models for the rotational braking, filling the magnetosphere of Bp stars, and chemical peculiarity.

Acknowledgements. This work was supported by grant GA ČR 13-10589S.

\section{References}

Abbott, D. C. 1979, in Mass loss and evolution of O-type stars (Dordrecht: D. Reidel Publishing Co.), IAU Symp., 83, 237

Abbott, D. C. 1980, ApJ, 242, 1183

Abbott, D. C. 1982, ApJ, 259, 282

Asplund, M., Grevesse, N., Sauval, A. J., \& Scott, P. 2009, ARA\&A, 47, 481

Babel, J. 1995, A\&A, 301, 823

Babel, J. 1996, A\&A, 309, 867

Bohlender, D. A., Rice, J. B., \& Hechler, P. 2010, A\&A, 520, A44

Bouret, J.-C., Lanz, T., Hillier, D. J., et al. 2003, ApJ, 595, 1182

Briquet, M., Aerts, C., Lüftinger, T., et al. 2004, A\&A, 413, 273

Castor, J. I., Abbott, D. C., \& Klein, R. I. 1975, ApJ, 195, 157

Cohen, D. H., Kuhn, M. A., Gagné, M., Jensen, E. L. N., \& Miller, N. A. 2008, MNRAS, 386, 1855

de Jager, C., Nieuwenhuijzen, H., \& van der Hucht, K. A. 1988, A\&AS, 72, 259

Ekström, S., Georgy, C., Eggenberger, P., et al. 2012, A\&A, 537, A146

Feldmeier, A., \& Shlosman, I. 2000, ApJ, 532, L125

Feldmeier, A., Puls, J., \& Pauldrach, A. W. A. 1997, A\&A, 322, 878

Gayley, K. G. 1995, ApJ, 454, 410

Gayley, K. G., \& Owocki, S. P. 1994, ApJ, 434, 684

Gvaramadze, V. V., Langer, N., \& Mackey, J. 2012, MNRAS, 427, L50

Harmanec, P. 1988, Bull. Astron. Inst. Czechosl. 39, 329

Hubený, I., Harmanec, P., \& Štefl, S. 1986, Bull. Astron. Inst. Czech., 37, 370

Huenemoerder, D. P., Oskinova, L. M., Ignace, R., et al. 2012, ApJ, 756, L34

Hummer, D. G., Berrington, K. A., Eissner, W., et al. 1993, A\&A, 279, 298

Hunger, K., \& Groote, D. 1999, A\&A, 351, 554

Hunger, K., Heber, U., \& Groote, D. 1989, A\&A, 224, 57

Kellett, B. J., Graffagnino, V., Bingham, R., Muxlow, T. W. B., \& Gunn, G. A. 2007 [arXiv: astro-ph/0701214]

Khokhlova, V. L., Vasilchenko, D. V., Stepanov, V. V., \& Romanyuk, I. I. 2000, Astron. Lett., 26, 177

Krtička, J. 2006, MNRAS, 367, 1282

Krtička, J., \& Kubát, J. 2009a, A\&A, 493, 585

Krtička, J., \& Kubát, J. 2009b, MNRAS, 394, 2065

Krtička J., \& Kubát J. 2010, A\&A, 519, A50

Krtička, J., Kubát, J. 2012, MNRAS, 427, 84

Krtička, J., Kubát, J., \& Groote, D. 2006, A\&A, 460, 145

Kubát, J., Puls, J., \& Pauldrach, A. W. A. 1999, A\&A, 341, 587

Kupka, F., Piskunov, N. E., Ryabchikova, T. A., Stempels, H. C., \& Weiss, W. W. 1999, A\&AS, 138, 119 
Kuschnig, R., Ryabchikova, T. A., Piskunov, N. E., Weiss, W. W., \& Gelbmann, M. J. 1999, A\&A, 348, 924

Lamers, H. J. G. L. M., \& Morton, D. C. 1976, ApJS, 32, 715

Lamers, H. J. G. L. M., Snow, T. P., \& Lindholm, D. M. 1995, ApJ, 455, 269

Landstreet, J. D., \& Borra, E. F. 1978, ApJ, 224, L5

Landstreet, J. D., Dolez, N., \& Vauclair, S. 1998, A\&A, 333, 977

Lanz, T., \& Hubeny I. 2003, ApJS, 146, 417

Lanz, T., \& Hubeny, I. 2007, ApJS, 169, 83

Lehmann, H., Tkachenko, A., Fraga, L., Tsymbal, V., \& Mkrtichian, D. E. 2007, A\&A, 471, 941

Leto, P., Trigilio, C., Buemi, C. S., Umana, G., \& Leone, F. 2006, A\&A, 458, 831

Lucy, L. B. 2012, A\&A, 544, A120

Lucy, L. B., \& White, R. L. 1980, ApJ, 241, 300

Marcolino, W. L. F., Bouret, J.-C., Martins, F., et al. 2009, A\&A, 498, 837

Martins, F., Schaerer, D., Hillier, D. J., et al. 2005, A\&A, 441, 735

Meynet, G., \& Maeder, A. 2006, Stars with the B[e] Phenomenon, eds. M. Kraus, \& A. S. Miroschnichenko, ASP Conf. Ser., 355, 27

Meynet, G., Ekström, S., \& Maeder, A. 2006, A\&A, 447, 623

Michaud, G. 2004, in The A-Star Puzzle, eds. J. Zverko, J. Žižňovský, S. J. Adelman, \& W. W. Weiss (Cambridge: Cambridge Univ. Press), IAU Symp., 224,173

Michaud, G., Richer, J., \& Vick, M. 2011, A\&A, 534, A18

Mihalas, D., Kunasz, P. B., \& Hummer, D. G. 1975, ApJ, 202, 465

Mikulášek, Z., Krtička, J., Henry, G. W., et al. 2011, A\&A, 534, L5

Molnar, M. R. 1973, ApJ, 179, 527

Najarro, F., Hanson, M. M., \& Puls, J. 2011, A\&A, 535, A32

Neiner, C., Landstreet, J. D., Alecian, E., et al. 2012, A\&A, 546, A44

Oskinova, L. M., Todt, H., Ignace, R., et al. 2011, MNRAS, 416, 1456

Owocki, S. P., \& Puls, J. 2002, ApJ, 568, 965

Owocki, S. P., \& ud-Doula, A. 2004, ApJ, 600, 1004

Owocki, S. P., Castor, J. I., \& Rybicki, G. B. 1988, ApJ, 335, 914

Owocki, S. P., Cranmer, S. R., \& Gayley, K. G. 1996, ApJ, 472, L115

Palla, F., \& Stahler, S. W. 1992, ApJ, 392, 667

Pauldrach, A. W. A., Hoffmann, T. L., \& Lennon, M. 2001, A\&A, 375, 161
Peterson, D. M. 1970, ApJ, 161, 685

Petit, V., Owocki, S. P., Wade, G. A., et al. 2013, MNRAS, 429, 398

Piskunov, N. E., Kupka, F., Ryabchikova, T. A., Weiss, W. W., \& Jeffery, C. S. 1995, A\&AS, 112, 525

Puls, J., Springmann, U., \& Lennon, M. 2000, A\&AS, 141, 23

Puls, J., Vink, J. S., \& Najarro, F. 2008, A\&ARv, 16, 209

Pyper, D. M., Ryabchikova, T., Malanushenko, V., et al. 1998, A\&A, 339, 822

Rivinius, T., Townsend, R. H. D., Kochukhov, O., et al. 2013, MNRAS, 429, 177

Romanyuk, I. I. 2007, Astrophys. Bull., 62, 62

Seaton, M. J., Zeippen, C. J., Tully, J. A., et al. 1992, Rev. Mex. Astron. Astrofis., 23,19

Shore, S. N., \& Brown, D. N. 1990, ApJ, 365, 665

Slettebak, A. 1994, ApJS, 94, 163

Springmann, U. W. E., \& Pauldrach, A. W. A. 1992, A\&A, 262, 515

Sundqvist, J. O., Puls, J., Feldmeier, A., \& Owocki, S. P. 2011, A\&A, 528, A64

Šurlan, B., Hamann, W.-R., Kubát, J., Oskinova, L., \& Feldmeier, A. 2012, A\&A, 541, A37

Townsend, R. H. D., \& Owocki, S. P. 2005, MNRAS, 357, 251

Townsend, R. H. D., Owocki, S. P. \& Groote, D. 2005, ApJ, 630, L81

Townsend, R. H. D., Oksala, M. E., Cohen, D. H., Owocki, S. P., \& ud-Doula, A. 2010, ApJ, 714, 318

Trigilio, C., Leto, P., Leone, F., Umana, G., \& Buemi, C. S. 2000, A\&A, 362, 281

Tsymbal, V., \& Mkrtichian, D. E. 2007, A\&A, 471, 941

ud-Doula, A., Owocki, S. P., \& Townsend, R. H. D. 2009, MNRAS, 392, 1022

Unglaub, K. 2008, A\&A, 486, 923

Vauclair, S. 1975, A\&A, 45, 233

Vauclair, S., Dolez, N., \& Gough, D. O. 1991, A\&A, 252, 618

Vick, M., Michaud, G., Richer, J., \& Richard, O. 2011, A\&A, 526, A37

Vink, J. S., de Koter, A., \& Lamers, H. J. G. L. M. 2001, A\&A, 369, 574

Votruba, V., Feldmeier, A., Kubát, J., \& Rätzel, D. 2007, A\&A, 474, 549

Yoon, S.-C., Langer, N., \& Norman, C. 2006, A\&A, 460, 199

Zboril, M., North, P., Glagolevskij, Yu. V., \& Betrix, F. 1997, A\&A, 324, 949 